\title{
MONITORING OBSERVATIONS OF METEOR ECHO AT THE EKB ISTPSB RAS RADAR: ALGORITHMS, VALIDATION, STATISTICS
}

\author{
R.R. Fedorov \\ Institute of Solar-Terrestrial Physics SB RAS, \\ Irkutsk, Russia,fedrr@isz.irk.ru
}

\author{
O.I. Berngardt \\ Institute of Solar-Terrestrial Physics SB RAS, \\ Irkutsk, Russia,berng@iszf.irk.ru
}

\begin{abstract}
The paper considers the implementation of algorithms for automatic search for signals scattered by meteor trails according to EKB ISTP SB RAS radar data. In general, the algorithm is similar to the algorithms adopted in specialized meteor systems. The algorithm is divided into two stages: detecting a meteor echo and determining its parameters. We show that on the day of the maximum Geminid shower, December 13, 2016, the scattered signals detected by the algorithm are foreshortening and correspond to scattering by irregularities extended in the direction of the meteor shower radiant. This confirms that the source of the signals detected by the algorithm is meteor trails. We implement an additional program for indirect trail height determination. It uses a decay time of echo and the NRLMSIS-00 atmosphere model to estimate the trail height. The
\end{abstract}

dataset from 2017 to 2019 is used for further testing of the algorithm. We demonstrate a correlation in calculated Doppler velocity between the new algorithm and FitACF. We present a solution of the inverse problem of reconstructing the neutral wind velocity vector from the data obtained by the weighted least squares method. We compare calculated speeds and directions of horizontal neutral winds, obtained in the three-dimensional wind model and the HWM-14 horizontal wind model. The algorithm allows real-time scattered signal processing and has been put into continuous operation at the EKB ISTP SB RAS radar.

Keywords: meteor trails, HF radar, atmosphere dynamics, automatic detection.

\section{INTRODUCTION}

Studies of meteor showers are relevant for dealing with asteroid and comet impact hazards. The study of meteor showers and individual meteors allows us to examine the relationship between meteor showers and parent bodies (asteroids and comets), and thus to study the dynamics of small bodies in the Solar System. In terms of upper atmosphere physics, meteors are considered to be sources of disturbances of the background atmosphere and provide insight into the generation of small-scale irregularities, as well as into their dynamics under the influence of background processes in the upper atmosphere. One of the most common methods of using meteors for diagnosing the upper atmosphere is to study the neutral wind speed. Meteor ablation occurs at altitudes of 60 to $150 \mathrm{~km}$, depending on the meteoroid mass, its velocity, etc. [McKinley, 1961; Briczinski et al., 2009]. Attendant processes - from airglow and ionization (for small meteors) to electrophonic effect and shock wave (for large fireballs) — are multiple and complex [Janches et al., 2009; Berngardt et al., 2013] and are under study [Zhu et al., 2016]. Massive meteoroids can ablate only partially, reaching Earth's surface. Smaller meteors ablate in the atmosphere almost completely, allowing us to determine the meteoroid mass from the ablation dynamics.

One of the popular methods for observing meteors are radar observations. In radar observations, two types of scattered signal are identified according to the form of a scattering object: scattering by increased ionization zone near a meteoroid (head echo) [Li et al., 2020] and scattering by meteor trail (trail echo). Meteor trails are extended ionized irregularities in the lower ionosphere (at altitudes below $150 \mathrm{~km}$ ), scattering by which is aspect [McKinley, 1961], therefore their observation requires a relatively low potential of a radar. Geometry of these observations is presented in Figure 1, $a$.

Scattering by a near-meteoroid region is more isotropic, its scattering cross-section is smaller than that of the scattering by a meteor trail, hence the need for sufficiently high-potential radars to study the head echo. Among such high potential radars are the radars MU [Kero et al., 2012], ALTAIR [Close et al., 2002], as well as most incoherent scatter radars (Millstone Hill [Erickson et al., 2001], EISCAT [Szasz et al., 2008], Arecibo [Mathews et al., 2003]). Head-echo studies facilitate the solution of the trajectory problems related to meteor showers and their sources. In this case, we can experimentally determine the body trajectory, atmospheric entry speed, and deceleration rate [Mathews et al., 2003, 2010; Szasz et al., 2008], which allows us to estimate its mass and the radiant direction.

Another method of analyzing the radiant deals with aspect characteristics of the meteor trail [Lovell, 1954; Jones et al., 2005; Campbell-Brown, 2008]. According to the ratio of plasma frequency of ionized meteor trail to sounding frequency, meteor trails are generally classified as underdense and overdense [Parris, 2003], with less massive meteoroids in the former group and more massive meteoroids in the latter. There is also an intermediate type of meteor trails, usually omitted by SuperDARN radars.

Obviously, there are a larger number of less massive meteors and therefore they are more often used in applied tasks of the upper atmosphere research. The trail formed after ablation of such a meteor is an elongated 
A)

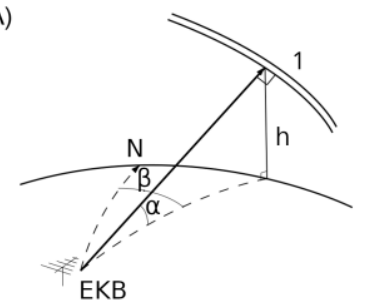

C)

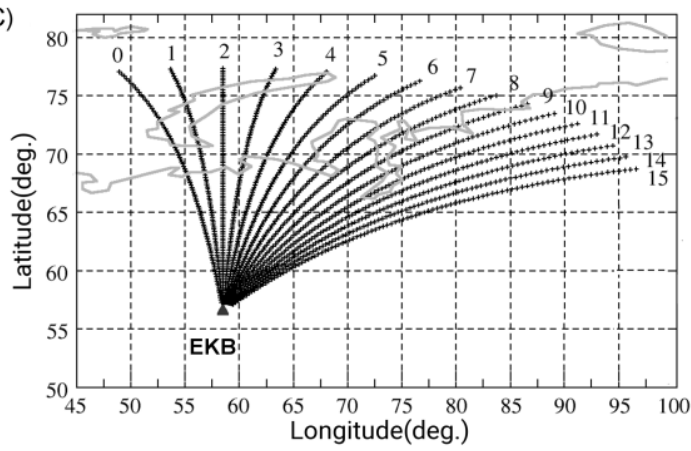

Figure 1. Scheme of backscattering by meteor trail MT $(a)$ : $\alpha$ and $\beta$ are the elevation angle and the azimuth respectively. EKB radar radiation patterns $(b)$ : $\mathrm{A}$ and $\mathrm{B}$ are for scanning by any possible beam; $\mathrm{C}$ is for radiation exactly perpendicular to the antenna array. The EKB radar field of view (c); numbers indicate ordinal numbers of beams

ionized region that, on the one hand, recombines and, on the other, spreads out due to ambipolar diffusion [Jones, Jones, 1990], therefore the electron density in the trail decreases with time. As a result, this scattering by the underdense trail is characterized by an exponential decay of the received signal power. The decay rate is related to the diffusion coefficient, which allows this coefficient to be measured directly. Furthermore, the trail is in a sufficiently dense atmosphere, therefore it is carried away by the neutral wind due to ion-neutral collisions. This enables measurements of neutral wind at heights of observation of meteor trails [Nakamura et al., 1991; Hall et al., 2006] and, when using dense networks of instruments such as meteor radar networks [Deegan et al., 1970] or SuperDARN radars [Hall et al., 1997], realtime monitoring of the motion of the upper atmosphere in a large spatial region.

An important task of monitoring meteor studies is to develop algorithms of automatic detection and determination of meteor echo parameters. Algorithms for examining scattering by meteor trails usually involve analyzing the variation of received signal power and searching for sharp peaks with an exponential decay, which is a signature of the underdense echo [Tsutsumi et al., 1999]. SuperDARN radars can detect the meteor echo both from mean correlation characteristics of the signal [Jenkins, Jarvis, 1999] and by analyzing the time dependence of amplitude of single pulse in the high sampling rate mode [Parris, 2003] or IQcomponents of the scattered signal representing a complex pulse sequence [Yukimatu, Tsutsumi, 2002].

This paper delves into the implementation of the algorithm for automatic meteor detection by the EKB ISTP SB RAS radar from signal IQ-components; addresses the inverse problem of reconstructing the neutral wind velocity vector from the findings, and validates these algorithms.

\section{EKB ISTP SB RAS RADAR}

The EKB ISTP SB RAS radar is a CUTLASS-type decameter over-the-horizon radar, designed and manufactured at the University of Leicester (UK), analogous to SuperDARN radars. The radar is located in the Sverdlovsk Region, Russia $\left(56.5^{\circ} \mathrm{N}, 58.5^{\circ} \mathrm{E}\right)$. The radar's field of view $\sim 52^{\circ}$ wide in azimuth is divided into 16 directions (beams) $3^{\circ}-6^{\circ}$ wide, depending on sounding frequency. The central axis of its field of view has a $19^{\circ}$ azimuth. The radar's field of view and orientation of its beams are shown in Figure 1, $b$. The radar works in a frequency range $8-20 \mathrm{MHz}$, ensuring a range resolution $15-45 \mathrm{~km}$ in standard modes and a maximum range $3000-4500 \mathrm{~km}$. The EKB radar is a stereoradar, i.e. it can run at two spaced carrier frequencies and different beams simultaneously. Its antenna array is linear and has a significant back lobe in the most commonly used frequency band 10-12 MHz, so the radiation pattern of each beam can be regarded as a cone surface. The characteristic radiation pattern for different beams is shown in Figure $1, b$.

The standard operating mode of the radar is radiation of multipulse (Golomb) sequences [Berngardt et al., 2015], providing both high spatial and spectral resolution. Subsequent autocorrelation signal processing, autocorrelation function (ACF) integration time (about 4-6 s) for each selected beam, and the analysis of the amplitudephase structure of the averaged ACF allow us to identify average characteristics of signals scattered by both ionospheric and ground surfaces [Ribeiro et al., 2013].

The most common meteor echo data processing approach in SuperDARN is to analyze characteristics of averaged ACFs: power, range, Doppler velocity along the line of sight, and spectral width. The selection is made by statistical thresholds of these parameters, determined experimentally from joint measurements with meteor radars. Time resolution of these techniques corresponds to the ACF integration time and is typically 4 $6 \mathrm{~s}$ [Arnold et al., 2003]. There are also algorithms that use raw IQ-components for complete cycle of detection and identification of scattering parameters [Yukimatu, Tsutsumi, 2002], or for preliminary selection of trailecho with subsequent determination of the scattering parameters from FitACF data [Parris, 2003].

\section{ALGORITHM FOR SEARCHING AND ESTIMATING METEOR- ECHO PARAMETERS}

\section{General description of the algorithm}

As previously indicated, the radar performs standard measurements, using multipulse complex sounding sequences allowing it to be regarded as a pulse radar with $0.3 \mu$ s duration of sounding signal and with nonperiodic pulse repetition. Time resolution of the radar is variable with a minimum repetition period of $2.4 \mu \mathrm{s}$ (minimum interpulse interval) in standard modes. Scat- 
tered signal IQ-components are recorded after the beginning of each sounding sequence with a sampling period of $0.3 \mu \mathrm{s}$, thereby ensuring a range measurement resolution of $45 \mathrm{~km}$.

The pulse mode of radar operation and the large range of distances pose a problem of cross-range interference (CRI) occurring when a power signal from a greater range from one pulse overlaps a signal of the subsequent pulse, and vice versa [Yukimatu, Tsutsumi, 2002]. The method of CRI rejection, described in detail in [Yukimatu, Tsutsumi, 2002], has been adopted in our algorithm to further refine the findings.

Processing of meteor signals involves two sequential tasks: 1) detecting a scattered signal and determining the distance to the scatter; 2) identifying scattered signal parameters: amplitude, decay time, Doppler velocity. Discuss the implementation of these tasks with the EKB radar.

\section{Detection of a candidate for meteor scattering}

A received signal is recorded as quadrature components, however, because we examine amplitude (peak power, decay time) and phase (Doppler velocity) signal parameters independently, it is more convenient to turn to amplitude $A\left(R_{k}, t\right)$ and phase $\varphi\left(R_{k}, t\right)$ time dependences, where $R_{k}$ is the $k$-th radar range (in the receiving mode, the radar accumulates signal for a time $(100-300 \mu \mathrm{s})$, thereby breaking the time discrete $(15-45 \mathrm{~km})$ corresponding to the spatial resolution of the radar); $t$ is the time.

The meteor trail observation time may be as much as several seconds [Tsutsumi et al., 1999], therefore we have to examine several consecutive sounding sequences, the typical length of a sequence being $0.1-0.2 \mathrm{~s}$. We also have to account for non-equidistance of the applied pulse sequence. Thus, the time dependence is convenient to represent as a discrete sequence $t_{l, n}$, where $l$ is the pulse number within the sequence; $n$ is the sequence number. For example, $A\left(R_{10}, t_{3,4}\right)$ is the amplitude of the response from the third pulse of the forth sequence at the 10th range.

In order to be selected as a candidate for meteor scattering, a signal must satisfy the following conditions (test stages):

Stage 1. Sharp increase in amplitude. Meteor trails are relatively rare, comparatively short-lived and quasistationary-in-space objects. We are interested in sharp increases in amplitude from which we can confidently determine the other meteor-echo parameters - decay time and mean velocity, therefore our search will invoke a simple excess of the increase over the noise level. The radar range $R_{k}$ being independent of time $t_{l, n}$ within the trail decay time, we can search for a meteor scattering for each fixed range gate $R_{k}$ independently, given that the signal amplitude shortly exceeds the noise level by $6 \mathrm{~dB}$ :

$$
A\left(R_{k}, t_{0, n}\right)>M,
$$

where $M$ is the noise level measured by the radar before starting the scan cycle $+6 \mathrm{~dB}$.

All the points $A\left(R_{k}, t_{0, n}\right)$ that satisfy (1) are relatively sharp increases in amplitude and pass to the next stage of the analysis.

Stage 2. High spatial localization. Meteor trails are rare and highly spatially localized objects. That is why, for each sharp increase in amplitude found at Stage 1 we monitor fulfilment of the latter condition - spatial localization of the sharp increase in amplitude. To do this requires that the average level at ranges adjacent $\left(R_{k-1}\right.$ and $\left.R_{k+1}\right)$ to the desired one $\left(\mathrm{R}_{\mathrm{k}}\right)$, should not exceed the threshold value $\mathrm{M}$ :

$$
\frac{A\left(R_{k+1}, t_{0, n}\right)+A\left(R_{k-1}, t_{0, n}\right)}{2}<M
$$

All the points $A\left(R_{k}, t_{0},{ }_{n}\right)$ satisfying (2) are spatially localized sharp increases in amplitude and pass to the next stage of the analysis.

Stage 3. Monotonic decrease in signal amplitude. As already mentioned above, the main feature of the scattering by underdense trails is an exponential decrease in signal amplitude with time. Accordingly, when detecting the meteor echo, we are looking for a number of increases with amplitude monotonically decreasing with time. The presence of noise and random amplitude variations hinder the work of this algorithm, therefore we analyze the points that are separated by a sufficiently large period of time in which the noise-like effects are much weaker than the main monotone decreasing. This characteristic gap in our algorithm is taken to be equal to the length of the sounding sequence, which is from 100 to $200 \mu \mathrm{s}$, depending on the type of sequence. We thus select by time $t_{0, n+l}$ the responses from the first pulses of the sequences following the sharp increase in amplitude detected at the previous stage. Of those sequences for which the amplitude of the signal from the first pulse satisfies condition (1) and whose first readings decrease with time every 100-200 $\mu$ s (we do not check satisfaction to condition (2)):

$$
A\left(R_{k}, t_{0, n+m}\right)>A\left(R_{k}, t_{0, n+m+1}\right), m=0 \ldots s-1,
$$

we form a set of $s$ sequences $S\left(R_{k}, t_{0, n}, s\right)$ used for further analysis as candidates for meteor scattering.

The formation of the set is completed if either condition (1) or condition (3) does not hold any more.

During the scanning, several such sets $S\left(R_{k}, t_{0, n}, s\right)$ can be formed, each then processed separately. In the current implementation of the algorithm, the sequences that fell into a set at this stage are not excluded from the analysis of the rest of the data and can then create another set.

To reduce the noise effect, from the resulting set $S\left(R_{k}, t_{0, n}, s\right)$ we exclude points not satisfying

$$
A\left(R_{k}, t_{l, n}\right)>M \text {. }
$$

Figure 2, a gives an example of an isolated amplitude sequence $A\left(R_{k}, t_{l, n}\right)$ for the fixed range gate $R_{k}$ corresponding to the meteor echo.

\section{Determination of scattered signal parameters}

The main parameters of underdense scattering by meteor trails are scattering point coordinates, the decay time, and the Doppler velocity along the line of sight. Knowing the scattering point coordinates is important for studies of diffusion processes in the upper atmosphere and the atmospheric wind fine structure at ablation altitudes. The time dependence of amplitude of backscattering by the underdense trail is primarily associated 

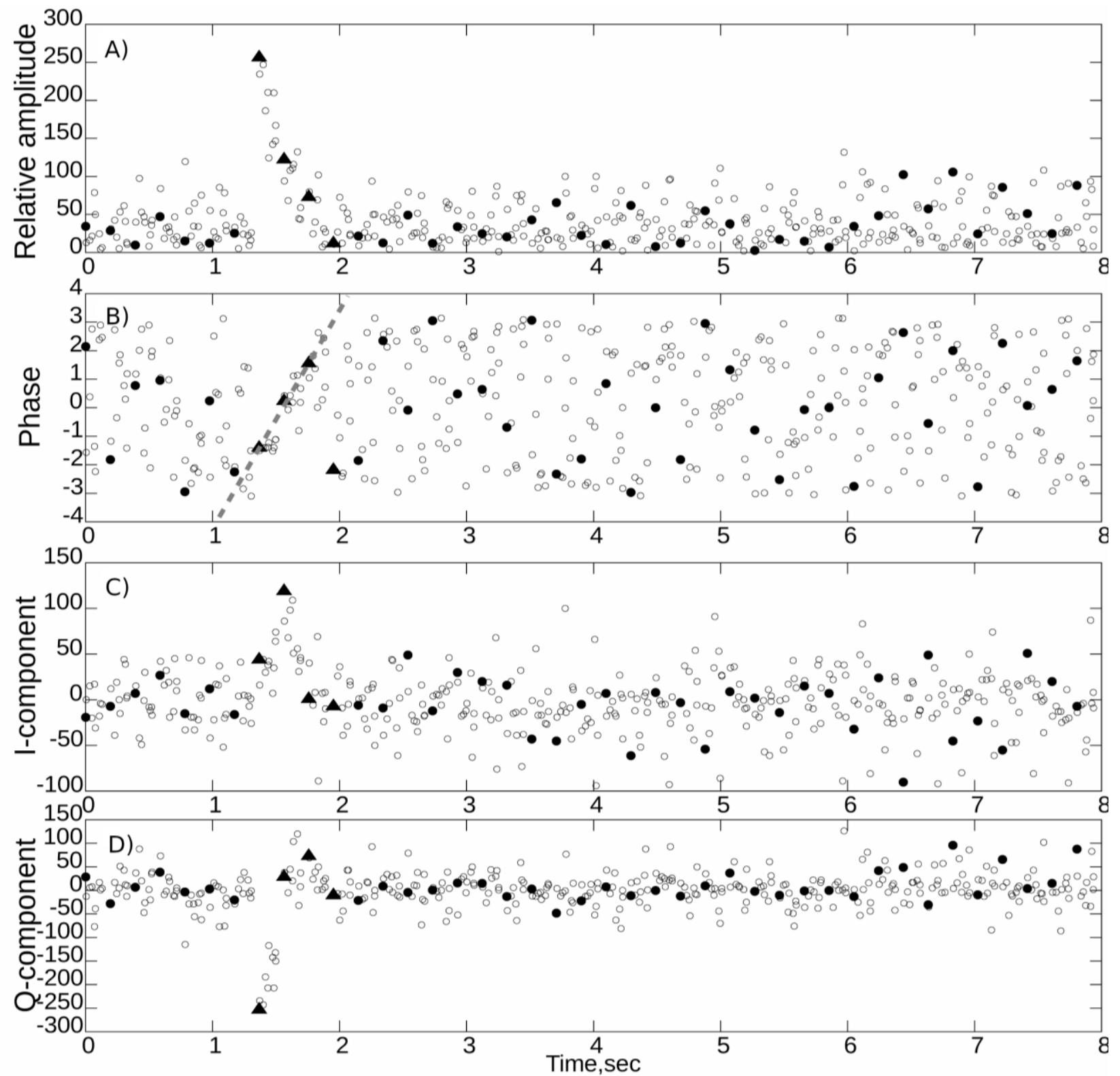

Figure 2. Time dependence of signal amplitude (a), phase (b), and IQ-components $(c, d)$ for the fixed range gate $R_{k}$ in the presence of meteor scattering. Black triangles denote the first pulses of the sequences identified in task 1; black circles mark the first pulses of the other sequences within the scan cycle; circles indicate a signal from all the rest of pulses

with the trail spreading out due to ambipolar diffusion, and hence the characteristic time of the exponential echo decay may be directly related to the diffusion coefficient [Jones, Jones, 1990]. The meteors observed by the EKB radar ablate at altitudes below $110 \mathrm{~km}$, where, on the one hand, the neutral component is dense enough to cause the ablation and, on the other hand, ions are not magnetized and their motion is generally controlled by the neutral wind. At altitudes above $110 \mathrm{~km}$ we omit, where the ionospheric plasma is magnetized, the drifts are determined by crossed electric and magnetic fields in the ionosphere. It is assumed that during the existence of the echo the speed and direction of the neutral wind cannot change significantly and the Doppler signal phase variation in a first approximation is linear, thereby allowing us to directly measure the wind speed along the line of sight [Tsutsumi et al., 1999] from the received signal phase change in the series of sounding sessions. Thus, the sequence of scattered signals satisfying requirements (1)-(3) is used to solve the inverse problem - to determine meteor echo characteristics. The problem is solved by the least squares method separately for the amplitude $A\left(R_{k}, t\right)$ and phase $\varphi\left(R_{k}, t\right)$ time dependences.

To simulate a signal, we employ the well-known model [Parris, 2003] of exponential amplitude decrease and linear phase variation:

$$
\left\{\begin{array}{l}
A_{m}(t)=\theta(t) A_{0} e^{-\frac{t}{\tau}} ; \\
\phi_{m}(t)=\theta(t)\left(s t+\phi_{0}\right) .
\end{array}\right.
$$

Here, $A_{0}$ is the initial amplitude of a scattered signal; $\tau$ is the decay time; $s$ is the linear phase shift corresponding to the Doppler freque 'ncy shift; $\varphi_{0}$ is the initial phase; $\theta(t)$ is the Heaviside step function (unit step function). 
Nonetheless, the sequences within one scan cycle are not time aligned, i.e. the delay between radiation of the last pulse of the preceding sequence and the first pulse of the subsequent one is a random variable and cannot be found by the existing control system of the EKB radar. As a result, between adjacent sequences that fall into the fitting set there is a phase shift undetectable by the system, which in the case of a set of two or more sequences leads to a dramatic deterioration in the quality of estimation of the Doppler velocity and to distortion in determining the decay time. Compensating for the hardware and software features of the EKB radar requires the model function to be updated. Since the delay between sequences is not defined, we can use only the mean value of the delay in the scan cycle.

Variations in the delay between the sequences being low, we ignore them when calculating the signal amplitude. To facilitate further calculations of the decay time, we logarithm the received signal amplitude, which allows us to find the amplitude from the known linear regression equation.

The phase under the assumption of linear phase incursion can be represented as a piecewise-defined set of lines of equal inclination with undefined initial phases. The modified signal model looks like:

$$
\left\{\begin{array}{l}
\ln \left(A_{m}\left(t_{l, n}\right)\right)=\ln \left(A_{0}\right)-\frac{t_{l, n}}{\tau} ; \\
\phi_{m}\left(t_{l, n}\right)=s t_{l, n}+\phi_{n},
\end{array}\right.
$$

where $l \in 0 \ldots N_{p}$ is the pulse number within the sequence; $n \in 0 \ldots N_{s}$ is the sequence number in the candidate set; $N_{p}$ is the number of pulses in the sequence; $N_{s}$ is the number of sequences in the candidate set.

The model parameters $\tau$ and $\left(s, \varphi_{n}\right)$ are searched using the method of least squares as an independent minimization of residual functions:

$$
\begin{aligned}
& \Omega_{\mathrm{a}}\left(A_{0}, \tau\right)= \\
& =\sum_{l=0}^{N_{p}} \sum_{n=0}^{N_{s}}\left|\ln \left(A_{0}\right)-\frac{t_{l, n}}{\tau}-\ln \left(A\left(R_{k}, t_{l, n}\right)\right)\right|^{2}=\min , \\
& \Omega_{\phi}\left(s, \phi_{0}, \ldots, \phi_{N_{s}}\right)= \\
& =\sum_{l=0}^{N_{p}} \sum_{n=0}^{N_{s}}\left|s t_{l, n}+\phi_{n}-\phi\left(R_{k}, t_{l, n}\right)\right|^{2}=\min .
\end{aligned}
$$

Due to the linearity of the model, from the desired parameters (initial amplitude $A_{0}$ and initial phases of segments $\varphi_{n}$, decay time $\tau$, and phase slope $s$ ) the problem is solved analytically.

\section{MODEL ADEQUACY}

Important issues are the adequacy of the model to experimental observations and the effect of noise on the accuracy of determining the parameters. A characteristic value allowing us to evaluate the adequacy of the model to experimental data is a residual between the model and the experiment. We have separately analyzed the distribution of the model residual by amplitude and phase from the dataset collected in 2016.

The normalized amplitude error in the solution was found from the standard deviation of the amplitude, obtained from the experimental data, from the model amplitude, normalized to the maximum amplitude of the signal scattered by the meteor trail, according to the expression

$$
\sigma_{A, r}=\frac{\sigma_{A}}{A\left(R_{k}, t_{0,0}\right)},
$$

where $\sigma_{A}$ is the standard deviation of the experimental amplitude from the model value:

$$
\sigma_{A}^{2}=\frac{1}{\left(N_{p} N_{s}-1\right)} \sum_{l=0}^{N_{p}} \sum_{n=0}^{N_{s}}\left(\phi_{m}\left(t_{l, n}\right)-\phi\left(R_{k}, t_{l, n}\right)\right)^{2} .
$$

The phase error in the solution was found from the standard deviation of the experimental phase from the model one:

$$
\sigma_{\phi}^{2}=\frac{1}{\left(N_{p} N_{n}-1\right)} \sum_{l=0}^{N_{p}} \sum_{n=0}^{N_{s}}\left(\phi_{m}\left(t_{l, n}\right)-\phi\left(R_{k}, t_{l, n}\right)\right)^{2} .
$$

The $\sigma_{A, r}$ and $\sigma_{\varphi}$ distributions experimentally measured from the data collected over 16 days corresponding to the passage of maxima of six meteoric showers between January and August 2016 are shown in Figure 3, $a$ and $b$ respectively. Selection of the meteor showers for the analysis allows us to effectively estimate the number of erroneously identified meteors and be more confident about the model validation results.

Referring to Figure 3, we can use two qualitative thresholds for further evaluation of the data validity:

$$
\left\{\begin{array}{l}
\sigma_{A, r}<0.5 \\
\sigma_{\phi}<0.7
\end{array}\right.
$$

The threshold for $\sigma_{A, r}$ restricts most distribution of the errors in approximation by the experimental amplitude model. The threshold for $\sigma_{\varphi}$ has been chosen for reasons of allowable error in determining the Doppler velocity: the deviation of $0.7 \mathrm{rad} / \mathrm{s}$ corresponds to an error in determining the meteor trail velocity of $\sim 2 \mathrm{~m} / \mathrm{s}$ for a frequency of $8 \mathrm{MHz}$, which is crucial for observations of the wind whose velocity is about $\pm 20 \mathrm{~m} / \mathrm{s}$. The observations having $\sigma_{\varphi}$ greater than the threshold do not meet our requirements to the accuracy in determining the velocity and are discarded. Figure $3, b$ shows that the introduction of the second threshold significantly (by $57 \%$ ) reduces the number of cases suitable for the interpretation. We omit the meteors corresponding to $\sigma_{\varphi}>0.7$.

The qualitative analysis has shown that the use of such confidence intervals can (due to excluding the cases when the model of underdense-trail scattering is inadequate for the experimental data) further limit the reliable identification of meteoric echo parameters by increasing the degree of confidence in the data acquired.

Examples of records satisfying both conditions (1)-(3) and qualitative fitting condition (9) are shown in Figure 4: $a, e$ and $c, g$ are signal amplitude and phase respectively; $b$, $f$ and $d, h$ are signal IQ-components. Black dots in the Figure represent a signal recording used for the fitting; circles show the points omitted as noise by criterion (4); 

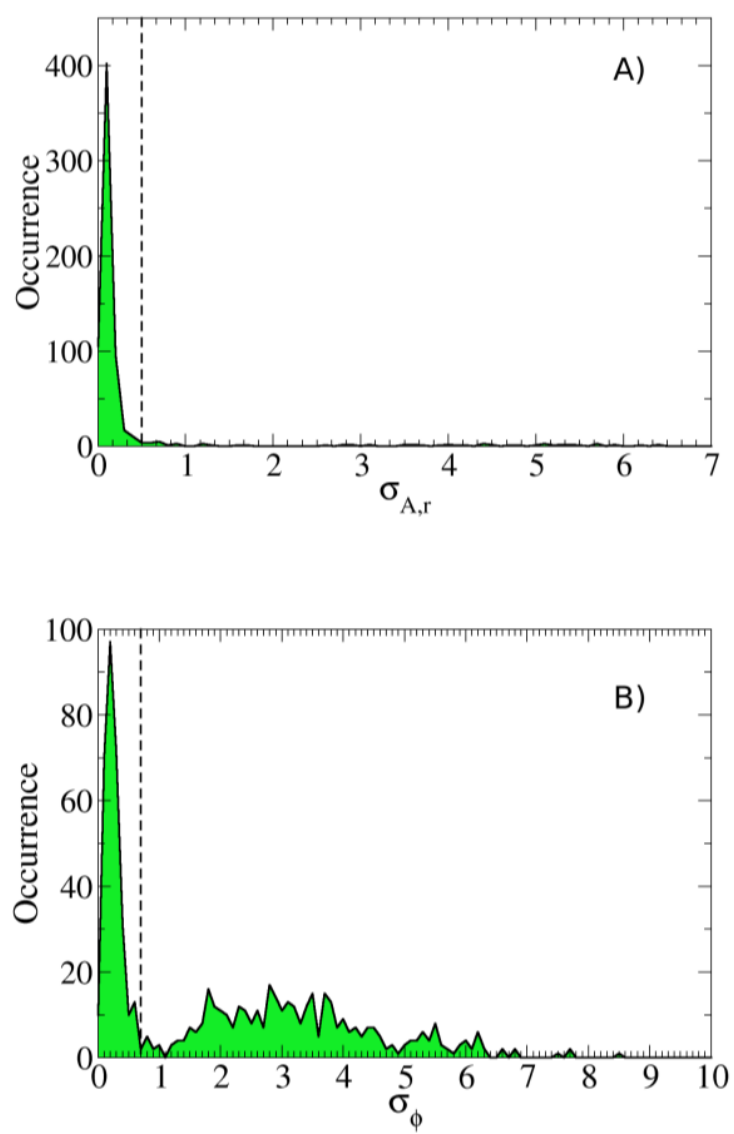

Figure 3. Distributions of a normalized amplitude error in the solution $\sigma_{, r}(a)$ and a phase error $\sigma_{\varphi}(b)$. The dashed line indicates threshold values

solid lines indicate the model that has, according to the fitting results, a minimum deviation from the experimental data.

One of the main geophysical parameters identified from the meteor-trail scattering is the diffusion coefficientthat can be estimated from the echo decay time [Jones, Jones, 1990]. For the underdense echo, the trail decay time in a first approximation is directly related to the diffusion coefficient at the height of ablation [Tsutsumi et al., 2009]:

$$
\tau=\frac{\lambda^{2}}{32 \pi^{2} D},
$$

where $\tau$ is the decay time; $\lambda$ is the probing signal wavelength; $D$ is the diffusion coefficient for this height. This expression is valid in the altitude range $85-95 \mathrm{~km}$ [Kam et al., 2019], which we will analyze below. In turn, the diffusion coefficient can be defined as follows:

$$
D=\frac{6.39 \cdot 10^{-2} K T^{2}}{p},
$$

where $T$ is the absolute temperature; $p$ is the pressure; $K$ is the zero field mobility of meteor ions (in such problems it is usually taken as $2.2 \cdot 10^{-4}\left[\mathrm{~m}^{2} \mathrm{~s}^{-1} \mathrm{~V}^{-1}\right]$ [Tsutsumi et al., 2009]).

The scattering altitude is calculated iteratively by the NRLMSIS-00 model, using the function of calculation of atmospheric parameters for given time, coordinates, and altitude. Figure 5 presents the distribution for scat- tering altitudes for each meteoric echo detected from January 01, 2017 to August 20, 2019. The results are based on the assumptions that the altitude of all meteor trails detected is related to the decay time by Equations (10), (11) and the error in determining the altitude is caused exclusively by inaccuracies in determining the diffusion coefficient. The simulation has shown that the error in determining the diffusion coefficient of $\pm 25 \%$ leads to an error in determining the altitude of about \pm 2 $\mathrm{km}$, which determines the accuracy of the results obtained in the first approximation. The decay time of some meteor trails may, however, be related to neutral atmosphere parameters in a more complicated manner - it is, for example, typical of the meteor trails of intermediate type or the meteor trails at altitudes above $95-100 \mathrm{~km}$ or below $85 \mathrm{~km}$. In future, to further data validation we may therefore apply other methods, e.g., elevation observations and observations of diurnal and semidiurnal variations in the neutral wind velocity.

Figure 5 indicates that the distribution is mostly in the range $80-100 \mathrm{~km}$, with a maximum at around 89 $\mathrm{km}$, which is consistent with the results obtained in [Tsutsumi et al., 2009]. Although in the data there is an altitude distribution cutoff due to the low potential of the radar, the meteor distributions obtained enable further analysis of the data in the altitude range $85-95 \mathrm{~km}$.

\section{Verification of aspect characteristics of the me- teor scattering in terms of maximum Geminid (2018) and Perseid (2019) showers}

To check the correctness of the interpretation of a scattered signal as scattering by an underdense meteor trail, we have examined aspect scattering. The current concept is that the meteoric echo during heavy meteor showers features a certain point on the celestial sphere, the radiant. Radiants of most showers can be calculated accurately enough for the day of maximum of shower and are published in astronomical calendars. The upper atmosphere being far above the height of meteor ablation, having a low density, and having little effect on the trajectory of meteors, their movement to the height of complete evaporation can be regarded as straight with a known direction, which can be calculated from the meteor radiant. Elongation of irregularity along the meteor trajectory and aspect characteristics of scattering by this elongated irregularity produce the known aspect dependence of meteor scattering [McKinley, 1961].

To make sure that the signals we detected are in fact the signals scattered by meteor trails, we checked for the aspect effect corresponding to meteor-shower scattering with a given radiant. To test the algorithm, we chose December 14, 2018 and August 13, 2019, the days with intense Geminid and Perseid meteor showers. Processing of experimental data from the EKB ISTP SB RAS radar revealed 2100 and 5400 reliable recordings of meteor scattering per day respectively.

For each meteor detected, we identified the geographical position of the scattering point from the observed azimuth and range to the meteor. The scattering altitude was determined from the decay time by the NRLMSIS-00 model, as described above. At the geographical scattering 

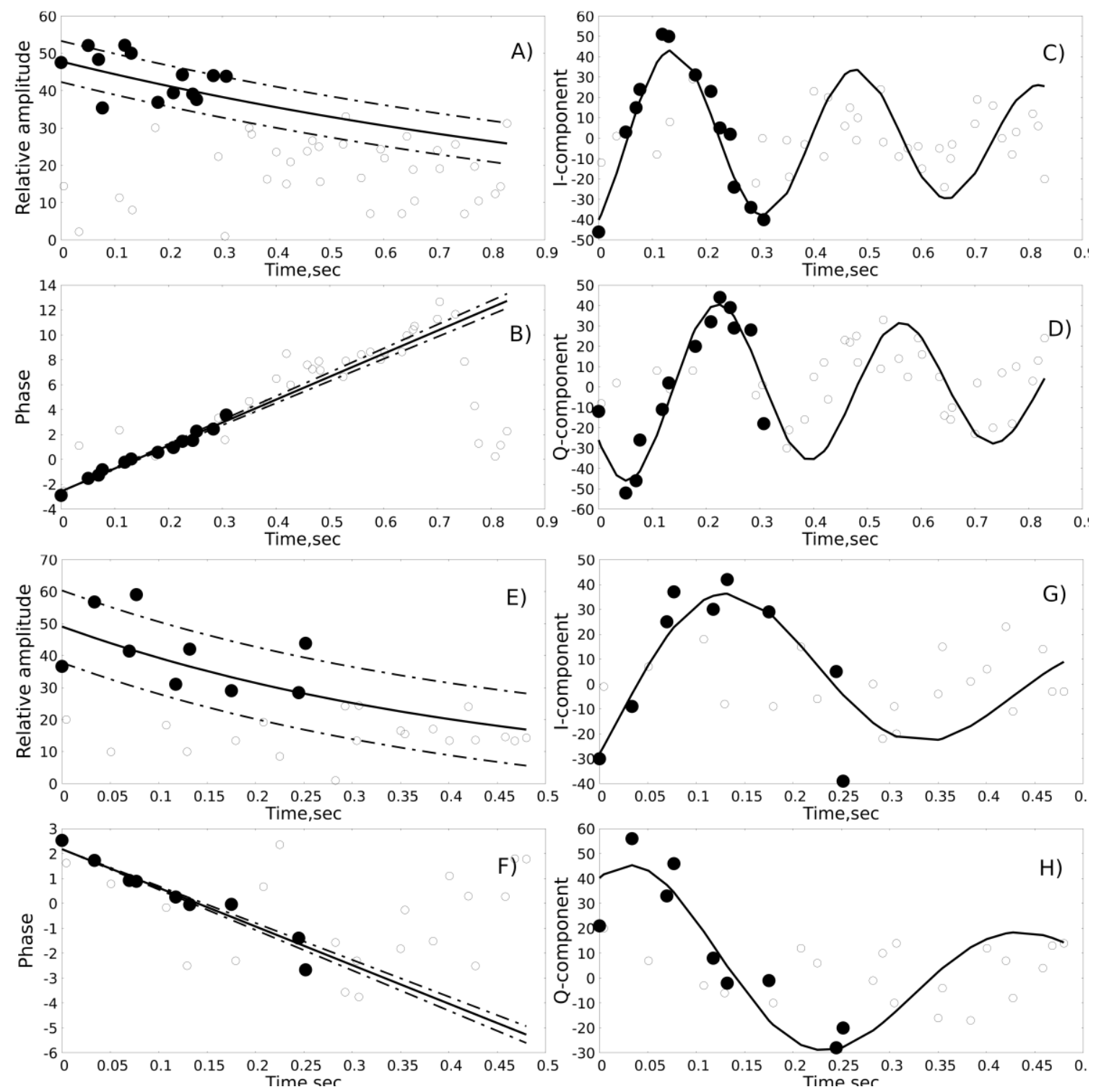

Figure 4. Examples of a qualitatively fitted meteor echo signal: $a-d-$ the first example; $e-h-$ the second example; $a, e-$ the signal amplitude modulus $A\left(R_{k}, t_{l, n}\right) ; c, g$ - the signal phase $\phi\left(R_{k}, t_{l, n}\right) ; b, d, f, h$ - signal IQ-components $I / Q$. Dots mark a received pilot signal; circles indicate noise measurements. Solid lines show model functions; dot-dash lines, SD

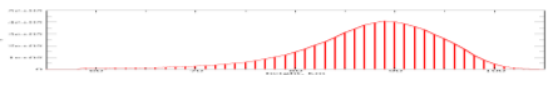

Figure 5. Distribution of estimated scattering altitude calculated from the decay time by the NRLMSIS-00 model. Observations from January 01, 2017 to August 20, 2019

point found, we calculated the direction vector to the meteor shower radiant and determined the angle between the line-of-sight direction to the meteor trail and the direction vector to the radiant. Accuracy in determining the azimuth direction to the trail is from $3^{\circ}$ to $6^{\circ}$, depending on the sounding frequency. The accuracy in determining the elevation angle direction to the trail depends on the accuracy in determining the scattering altitude and is linked with the accuracy in determining the decay time of the trail, the density of the neutral atmosphere, temperature, pressure, and many other parameters. When estimating the altitude determination accuracy, we hold that all these variations lead to a dif- fusion coefficient determination error of less than $20 \%$. This corresponds to an altitude determination accuracy of about $2 \mathrm{~km}$ and an elevation accuracy not lower than $0.3^{\circ}$. The simulation we have carried out has revealed that in terms of the deviation from the perpendicular to the trail direction the error is $\pm 2^{\circ}$ for Geminids and $\pm 1^{\circ}$ for Perseids. When calculating the direction to a meteor, we took into account the radiation pattern conicity associated with the linear antenna arrangement [Shepherd, 2017].

Since at the time of the experiment the EKB radar could not measure the scattered signal elevation angle, and its radiation pattern (RP) has a pronounced back lobe, this meteor might have been observed in the back lobe. When estimating the aspect angle, the calculations were therefore made for cases of detecting meteors both in the back and main lobes. Figure $6, a, b$ shows angle distributions between the direction to the radiant and the direction to the scattering point under the assumption of scattering in the main (green) and back (red) lobes. Figure 6, $a$ 
indicates that the scattering in the main lobe has essential aspect characteristics — most meteors are observed when the line of sight is almost perpendicular to the direction to the meteor radiant, but there is the second peak probably associated with the scattering in the back lobe. Accordingly, we estimate the distribution at the level of 0.707 , which yields a width of $21^{\circ}$. Figure $6, b$ displays a picture suggesting that meteors are generally observed in the back lobe, with a substantially greater width of distribution around $90^{\circ}$ : a width at the level of 0.707 is $33^{\circ}$. This leads to a preliminary conclusion that the scattering has aspect characteristics typical of meteor showers. A more detailed analysis requires interferometric observations made by the radar for effective identification of scattering in the front and back fields of view.

\section{Statistics of meteor trail observations with the EKB radar}

Figure 7 presents statistics of meteor observations made by the EKB radar over the period from late January 2017 to August 2019. The statistics includes over 6.8 million meteor observations, or about 260 meteors per hour, which is close to the results obtained by special-purpose devices [Premkumar et al., 2019; Korotyshkin et al., 2019]. Figure 7, $a$ shows the mean number of echo signals detected by the algorithm, depending on the local sidereal time (LST). We can see that the main peak occurs during the dawn hours; the minimum; at 18-19 LST. This distribution is similar to those obtained using both optical methods [Lovell, 1954] and other radar installations [Thomas et al., 1988]. Figure 7, $b$ illustrates the distribution of the number of meteor trails by the decay time. The Figure shows that the most probable decay time is around 0.6-0.7 s. Figure 7, $d$ depicts the distribution of the number of meteor trails by the Doppler velocity along the line of sight. The Figure suggests that the velocity in modulus does not usually exceed $150 \mathrm{~m} / \mathrm{s}$, thus implying that the search limits for the neutral velocity have also been chosen correctly. Figure 7, $c$ shows the distribution of the number of meteors by range. It indicates most meteor echo observations are centered at distances up to $300 \mathrm{~km}$, which corresponds to the known range restriction used for processing meteor data from SuperDARN radars [Jenkins et al., 1998].

To test the algorithm, we compared the calculated Doppler velocities determined by our algorithm and by the standard algorithm FitACF [Ribeiro et al., 2013]. The comparison was made at the points interpreted by the new algorithm as a meteor echo. Figure 8 compares Doppler velocity distributions. We can see the two algorithms are consistent, which allows us to use the new algorithm to identify neutral wind parameters at meteor ablation altitudes.

\section{DETERMINING THE FULL NEUTRAL WIND VELOCITY VECTOR}

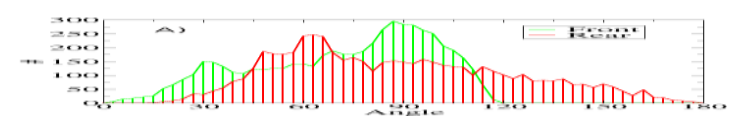

As shown previously, the new algorithm can determine the decay time and the Doppler velocity of a meteor trail. We have also demonstrated the possibility for determining the scattering altitude from the decay time, which allows us to find scattering point coordinates and location of the line of sight, even absent the interference grid, as in the case of the EKB radar until September 2019

Combination of these factors and the fact that the radar field of view is quite wide enable us to solve the problem of reconstructing the full vector of the neutral wind velocity under the assumption of wind isotropy in the field of view. In this case, we can find the full vector of the neutral wind velocity, using the weighted least squares method.

As such, the mean wind is determined from the experimental data given that the weighted quadratic residual functional is minimum:

$$
\begin{aligned}
& \Omega\left(V_{x}, V_{y}, V_{z}\right)=\sum_{i}\left\{W ( R _ { i } ) \left(V_{d, i}+V_{x} k_{x, i}+\right.\right. \\
& \left.\left.+V_{y} k_{y, i}+V_{z} k_{z, i}\right)^{2}\right\}=\min .
\end{aligned}
$$

Here $V_{d, i}$ is the measured velocity along the line of sight; $V_{x}, V_{y}, V_{z}$ are the neutral wind projections in the horizontal coordinate system that is local for the scattering point of the $i$-th meteor and locally parallel to Earth's surface under the approximation of spherical Earth (the $\mathrm{X}$-axis is directed to the north; the Y-axis, to the west; the Z-axis, vertically upward); $k_{x, i}, k_{y, i}, k_{z, i}$ are the unit vector components in the direction of the line of sight to the echo, which are recalculated into its local coordinate system; $R_{i}$ is the range from the radar to the scattering meteor trail; $i$ is the serial number of the meteor in the period of interest; $W\left(R_{i}\right)$ is the weight function. It is necessary to introduce the weight because of the great (up to 45 $\mathrm{km})$ sounding pulse length, which gives a large error in determining the position of the line of sight in space at short $(<300 \mathrm{~km})$ ranges, and hence a significant error in calculating horizontal components of the velocity vector. Referring to Figure 7, $c$, such meteor trails prevail.

The problem of determining the vector components by method (12) is a simple analytical problem that reduces to solving a system of three linear equations for $V_{x}, V_{y}$, $V_{z}$. The only problem to be solved is the correct definition of the weight function $W\left(R_{i}\right)$.

According to the principles of the weighted least squares method, at normal distribution of errors the weight function $W\left(R_{i}\right)$ is inversely proportional to the variance of the corresponding sum element:

$$
W\left(R_{i}\right)=\sigma_{V}^{-2}\left(R_{i}\right) .
$$

Thus, finding the weight $W\left(R_{i}\right)$ requires us to estimate the velocity variance as a function of distance to the meteor. In this case, when determining velocity vector components by a random parameter, the elevation angle $\alpha\left(R, h_{0}\right)$ remains which is uniquely determined from the scattering range $R$ and altitude $h_{0}$. At the same time, we assume radio signal propagation to be rectilinear. Refraction, especially at small ranges and relatively

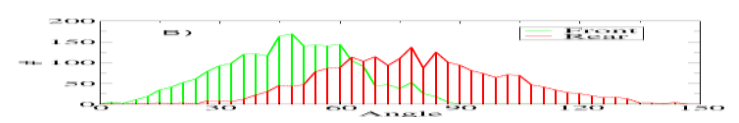

Figure 6. Distribution of angles between the direction to the radiant and the direction to the scattering point under the as- 
sumption of scattering in the main (green) and back (red) lobes: Geminids on December 14, 2018 (a); Perseids on August 13, $2019(b)$
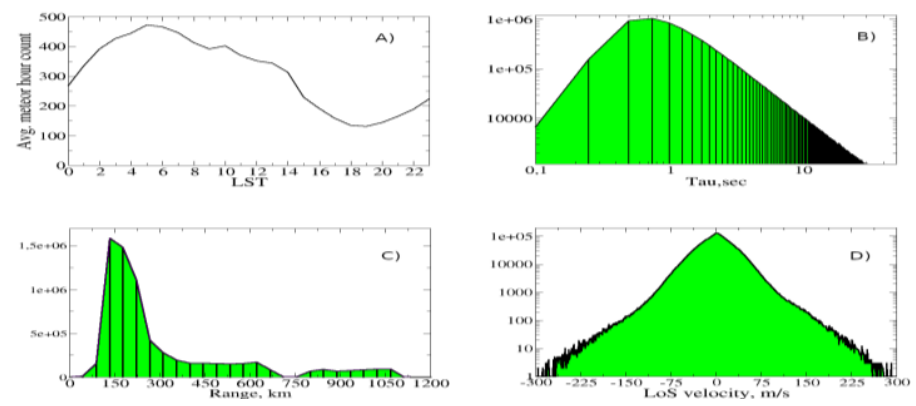

Figure 7. Statistics of meteor scattering observations with the EKB radar: $a$ - the mean number of meteors per hour, depending on the local sidereal time; $b$ - meteor trail decay time distribution; $c$ - distribution of the radar range to the trail; $d-$ distribution of the Doppler velocity along the line of sight

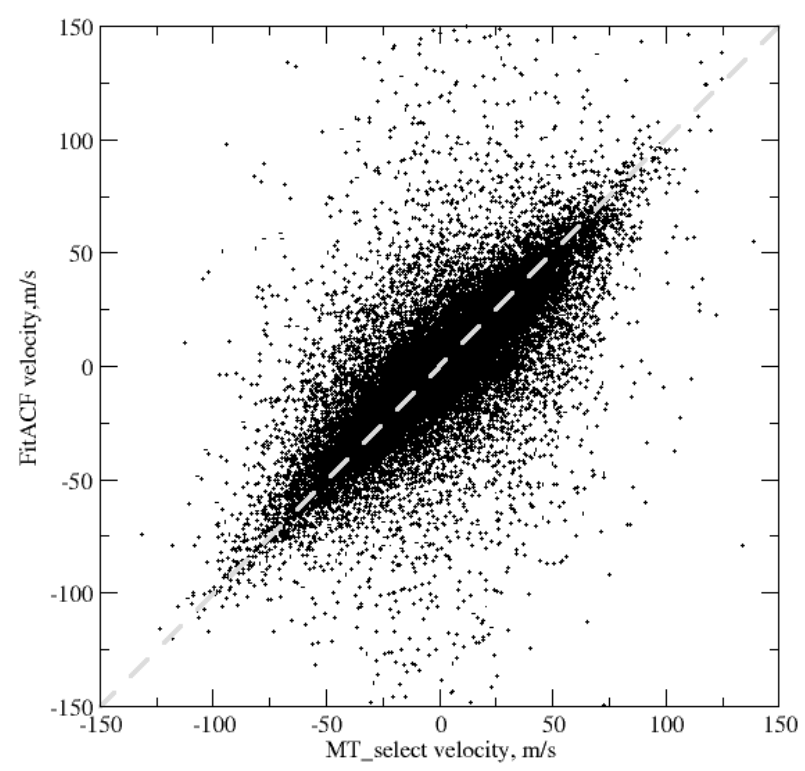

Figure 8. Comparison between Doppler velocities derived from the results obtained by the new algorithm and FitACF. The gray dashed line is the line $x=y$

high elevation angles, can be ignored in a first approximation, as the plasma frequency of the ionosphere at these altitudes is usually quite low - much lower than the operating sounding frequency. The parameter influencing the range is spatial resolution $\delta r$ providing uniform distribution of a possible range to a trail from $R$ to $R+\delta r$. Thus, we get uniform distribution for

$$
\alpha=a \cos \left(\frac{R_{\mathrm{E}}^{2}+R^{2}-\left(R_{\mathrm{E}}+h_{0}\right)^{2}}{2 R_{\mathrm{E}} R}\right)
$$

from $\alpha\left(R, h_{0}\right)$ to $\alpha\left(R+\delta r, h_{0}\right)$, where $R_{\mathrm{E}}$ is the Earth radius. Since the line-of-sight vector components directly depend on the elevation angle, the velocity variance is proportional to the elevation angle variance. Then, the weight of each point can be found as follows:

$$
W(R)=\frac{12}{\left(\alpha\left(R, h_{0}\right)-\alpha\left(R+\delta r, h_{0}\right)\right)^{2}} .
$$

It is Expression (15) that should be used when calculating the neutral wind velocity by weighted least squares method (12).

\section{Verification of the method for reconstructing the neutral wind velocity}

To test the method, we compared the findings with those obtained by the horizontal neutral wind model HWM-14. Observations performed from January 01, 2017 to August 20, 2019 have shown the mean number of 260 meteors per hour. For the analysis, we have used data for different seasons. The observations were divided into time intervals of $1 \mathrm{hr}$, used to determine the full wind velocity vector. The resulting horizontal components were averaged within the hour of interest over the entire observation period. In the same manner, we averaged the velocity vector components obtained by the HWM-14 model. As an example, Figure 9 shows the observational result obtained from January 8 to 25,2017 in the altitude range $85-95 \mathrm{~km}$. Panels $a$ and $b$ display calculated (black line) and model (gray line) meridional and zonal horizontal wind velocity components; panels $c$ and $d$ show SD of the meridional and zonal wind velocity components (gray line) and the distribution of the mean number of meteors per hour (dash-dot line); panels $e$ and $f$ compare the wind velocities calculated in threedimensional (black line) and two-dimensional (gray line) models. Panel $g$ depicts distribution of the vertical velocity according to the three-dimensional calculation.

As we can see in Figure 9, the calculated wind velocity components are qualitatively consistent with the model ones. Near the daily minimum of the number of meteors detected there is a significant increase in SD of the calculated velocity $(25-60 \mathrm{~m} / \mathrm{s})$. We can also see a significant SD $(15-20 \mathrm{~m} / \mathrm{s})$ of the calculated velocity, which is likely related to the fact that all the calculations are carried out under the assumption of scattering only in the front lobe, without elevation angle measurements. When testing this algorithm, we found a wide scatter of the calculated vertical component (SD $40 \mathrm{~m} / \mathrm{s}$ ), which is expected in the experiment due to small elevation angles observed. Meanwhile, the mathematical expectation of the calculated vertical component was close to zero, which is consistent with the known fact that the vertical wind velocity is low in this region. We therefore made the same calculation, excluding the vertical component (i.e. two-dimensional calculation), obtaining on average similar results of the 2D and 3D horizontal wind velocity calculations, which is attributed to the smallness of 
the mean vertical velocity.
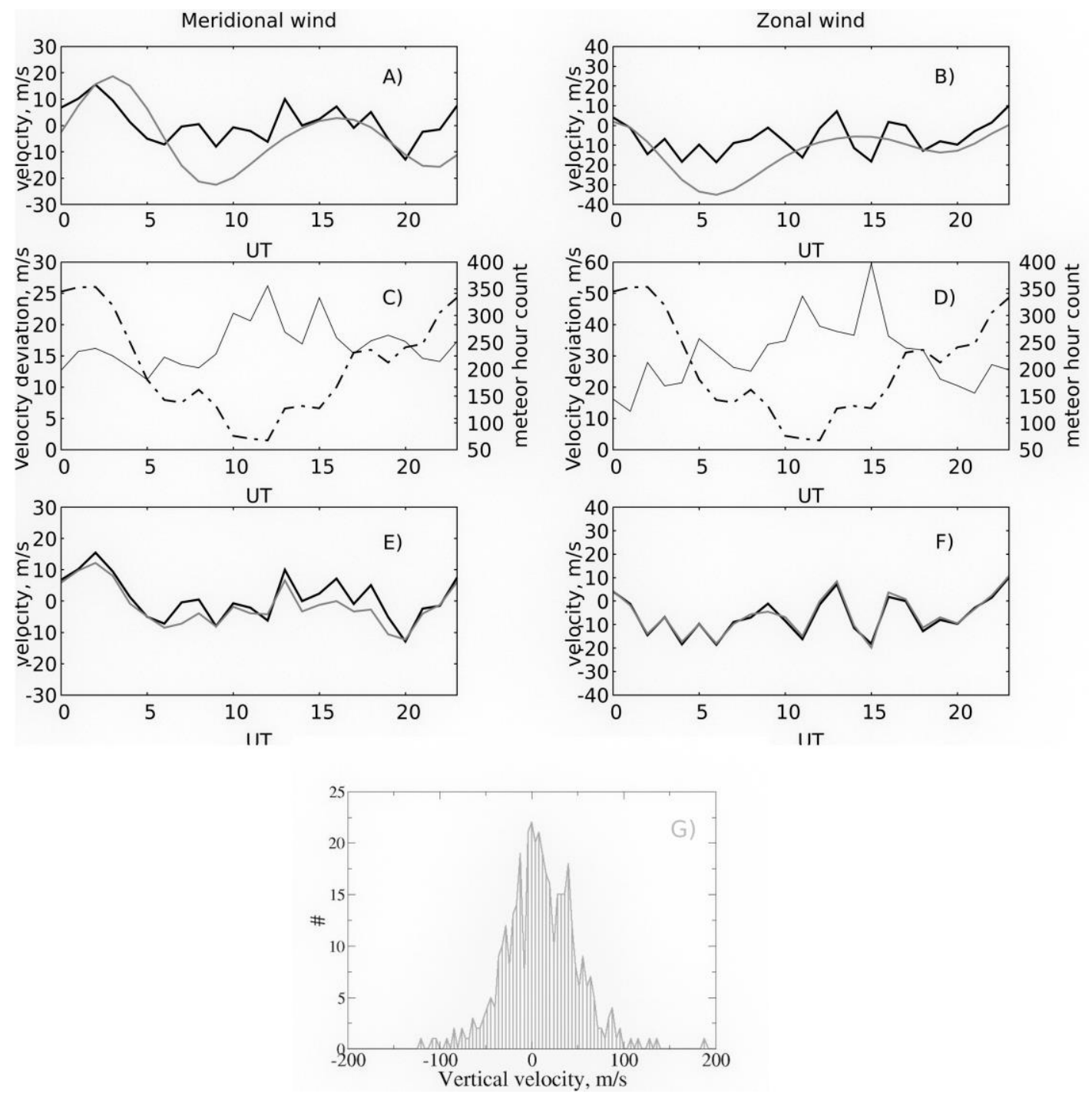

Figure 9. Comparison between the results obtained by the HWM-14 model and during the experiment in the altitude range 85$95 \mathrm{~km}: a$ and $b$ are meridional and zonal wind velocity components (positive values correspond to the northward and westward motion respectively), averaged by altitudes $(85-95 \mathrm{~km})$ and time; black lines indicate the experiment; gray lines, the HWM-14 model; $c$ and $d-\mathrm{SD}$ of the calculated meridional and zonal velocity components (gray lines) and the average number of meteors per hour (dash-dot line); $e$ and $f$ - comparison between 2D and 3D calculations: 3D (black line), 2D (gray line); $g$ - distribution of vertical velocities for 3D calculation. Observations from January 08, 2017 to January 25, 2017

\section{CONCLUSION}

The paper delves into the implementation of algorithms for automatic search for signals scattered by meteor trails, using the EKB ISTP SB RAS radar data. The algorithm consists of two stages: 1 ) detecting the meteor echo; 2) determining its parameters. The first stage involves searching for spatially localized sharp increases in amplitude over the average signal level, which monotonically decrease with time. At the second stage, the least squares method is used to approximate amplitude and phase time dependences by the exponential model of amplitude and the linear model of phase; and additional filtration of meteor trails detected is carried from the overall accuracy of phase and amplitude approximation. In general, the algorithm is similar to the algorithms applied in special-purpose meteor installations [Tsutsumi et al., 1999], but takes into account the hard- ware and software features of the EKB radar.

Testing the algorithm has shown that during days of maximum Geminid shower on December 14, 2019 and Perseid shower on August 13, 2019 the signals detected by the algorithm are foreshortening (see Figure 6, $a$ and $b$ ). In the case of Geminids, the aspect scattering corresponds to the assumption that meteor trails are generally observed in the main lobe, and the distribution width at the level of 0.707 is around $21^{\circ}$. For Perseids, the aspect scattering agrees with the assumption that meteors are largely observed in the back lobe, the distribution width at the 0.707 level is about $33^{\circ}$. It is impossible to separate the back and main lobes from the radar data in the reporting period because of the lack of sub-antennas in the array. This confirms that the source of signals detected by the algorithm with a high degree of probability is the scattering by meteor trails. We have also compared calculated Doppler 
velocities determined by our algorithm and by the standard algorithm FitACF (applied in SuperDARN radars to longitudinal velocity calculations [Ribeiro et al., 2013]). The comparison showed on average good agreement between the longitudinal velocities obtained by these methods.

The paper addresses the inverse problem of reconstructing the neutral wind velocity vector from the data obtained by the weighted least squares method under the assumption of isotropy of the neutral velocity field in the local coordinate system of the meteor. From geometric considerations, we have found the weight function of the method and have implemented the algorithm for determining the full vector of the neutral wind velocity under the assumptions described.

To test the method for solving the inverse problem of reconstructing the full vector of the neutral wind velocity from long-term meteor echo measurements (from January 01, 2017 to August 20, 2019), we have reconstructed velocities and directions of neutral winds in models of 2D (horizontal) and 3D wind. We have compared the findings with those obtained by the horizontal wind model HWM-2014. The computational scheme of wind velocity and direction (2D or 3D) have no significant effect on the daily average variation of winds. A more detailed comparison requires analysis of data on the additional (interference) antenna array, put into operation only recently, to separate scattering in the front and back lobes.

The algorithm for detecting signals scattered by meteor trails and for identifying parameters of these trails: longitudinal Doppler velocity and decay time, has been put into full time service at the EKB ISTP SB RAS radar.

The results were obtained using data from the EKB ISTP SB RAS radar. The results were obtained using the equipment of Shared Equipment Center «Angara» [http://ckp-angara.iszf.irk.ru]. The work was financially supported by the Ministry of Science and Higher Education of the Russian Federation.

\section{REFERENCES}

Arnold N.F., Cook P.A., Robinson T.R., Lester M., Chapman P.J., Mitchell N. Comparison of D-region Doppler drift winds measured by the SuperDARN Finland HF radar over an annual cycle using the Kiruna VHF meteor radar. Ann. Geophys. 2003, vol. 21, no. 10, pp. 2073-2082. DOI: 10.5194/angeo-21-2073-20033.

Berngardt O.I., Voronov A.L., Grkovich K.V. Optimal signals of Golomb ruler class for spectral measurements at EKB SuperDARN radar: Theory and experiment. Radio Sci. 2015, vol. 50, no. 6, 2014RS005589, pp. 486-500. DOI: 10.1002/ 2014 RS005589.

Berngardt O.I., Kurkin V.I., Zherebtsov G.A., Kusonski O.A., Grigorieva S.A. Ionospheric effects during first 2 hours after the "Chelyabinsk" meteorite impact. arXiv. 2013, arXiv: 1308.3918 [physics.geo-ph].

Briczinski S.J., Mathews J.D., Meisel D.D. Statistical and fragmentation properties of the micrometeoroid flux observed at Arecibo. J. Geophys. Res.: Space Phys. 2009, vol. 114, no. A4, A04311. DOI: 10.1029/2009JA014054.

Campbell-Brown M.D. High resolution radiant distribution and orbits of sporadic radar meteoroids. Icarus. 2008, vol. 196, no. 1, pp. 144-163. DOI: 10.1016/j.icarus.2008.02.022.
Close S., Hunt S.M, McKeen F.M., Minardi M.J. Characterization of Leonid meteor head echo data collected using the VHF-UHF Advanced Research Projects Agency Long-Range Tracking and Instrumentation Radar (ALTAIR). J. Geophys. Res. 2002, vol. 37, iss. 1, pp. 9-1. DOI: 10.1029/2000RS002602.

Deegan N.F., Fitzpatrick R., Forti G., Grossi M., Schaffner M., Southworth R. Study of meteor wind measurement techniques. V. 1. Final report. Contract AF 19(628)3248. Defense Technical Information Center, 1970. 128 p.

Erickson P.J., Lind F.D., Wendelken S.M., Faubert M.A. Meteor head echo observations using the Millstone Hill UHF incoherent scatter radar system. Meteoroids 2001 Conference. ESA Special Publ., 2001, vol. 495, pp. 457-463.

Hall G.E., MacDougall J.W., Moorcroft D.R., St.-Maurice J.P., Manson A.H., Meek C.E. Super Dual Auroral Radar Network observations of meteor echoes. J. Geophys. Res. 1997, vol. 102, iss. A7, pp. 14603-14614. DOI: 10.1029/97JA00517.

Hall C.M., Aso T., Tsutsumi M., Nozawa S., Meek C., Manson A. Comparison of meteor and medium frequency radar kilometer scale $\left\{\right.$ MLT\} dynamics at $70^{\circ}$ N. J. Atmos. Solar-Terr. Phys. 2006. vol. 68, no. 3-5, pp. 309-316. DOI: 10.1016/ j.jastp.2005.03.025.

Janches D., Dyrud L.P., Broadley S.L., Plane J.M.C. First observation of micrometeoroid differential ablation in the atmosphere. Geophys. Res. Lett. 2009, vol. 36, no. 6, L06101. DOI: 10.1029/2009GL037389.

Jenkins B., Jarvis M.J. Mesospheric winds derived from SuperDARN HF radar meteor echoes at Halley, Antarctica. Earth, Planets and Space. 1999, vol. 51, pp. 685689. DOI: $10.1186 / \mathrm{BF} 03353226$.

Jenkins B., Jarvis M.J., Forbes D.M. Mesospheric wind observations derived from Super Dual Auroral Radar Network (SuperDARN) HF radar meteor echoes at Halley, Antarctica: Preliminary results. Radio Sci. 1998, vol. 33, no. 4, pp. 957965. DOI: 10.1029/98RS01113.

Jones W., Jones J. Ionic diffusion in meteor trains. J. Atmos. Terr. Phys. 1990, vol. 52, pp. 185-191. DOI: 10.1016/00219169(90)90122-4.

Jones J., Brown P., Ellis K.J., Webster A.R., CampbellBrown M., Krzemenski Z., Weryk R.J. The Canadian Meteor Orbit Radar: system overview and preliminary results. Planet. Space Sci. 2005, vol. 53, pp. 413-421. DOI: 10.1016/j.pss. 2004.11.002.

Kam H., Kim Y.H., Mitchell N.J., Kim J.-H., Lee C. Evaluation of estimated mesospheric temperatures from 11-year meteor radar datasets of King Sejong Station $\left(62^{\circ} \mathrm{S}, 59^{\circ} \mathrm{W}\right)$ and Esrange (68 N, $21^{\circ}$ E). J. Atmos. Solar-Terr. Phys. 2019, vol. 196, pp. 105148. DOI: 10.1016/j.jastp.2019.105148.

Kero J., Szasz C., Nakamura T., Meisel D.D., Ueda M., Fujiwara Y., Terasawa T., Nishimura K., Watanabe J. The 2009-2010 MU radar head echo observation programme for sporadic and shower meteors: radiant densities and diurnal rates. Mon. Not. Royal Astron. Soc. 2012, vol. 425, no. 1, pp. 135-146. DOI: 10.1111/j.1365-2966.2012.21407.x.

Korotyshkin D., Merzlyakov E., Sherstyukov O., Valiullin F. Mesosphere/lower thermosphere wind regime parameters using a newly installed SKiYMET meteor radar at Kazan $\left(56^{\circ} \mathrm{N}, 49^{\circ} \mathrm{E}\right)$. Adv. Space Res. 2019, vol. 63, no. 7, pp. 2132-2143. DOI: 10.1016/j.asr.2018.12.032.

Li Y., Zhou Q., Scott M., Milla M. A Study on meteor head echo using a probabilistic detection model at Jicamarca. J. Geophys. Res.: Space Phys. 2020, vol. 125, no. 1, e2019JA027459. DOI: 10.1029/2019JA027459.

Lovell A.C.B. Meteor Astronomy. Oxford, Clarendon Press., 1954, 463 p.

Mathews J.D., Doherty J., Wen C.H., Briczinski S., Janches D., Meisel D. An update on UHF radar meteor obser- 
vations and associated signal processing techniques at Arecibo Observatory. J. Atmos. Solar-Terr. Phys. 2003, vol. 65, no. 10, pp. 1139-1149. DOI: 10.1016/j.jastp.2003.07.009.

Mathews J.D., Briczinski S.J., Malhotra A., Cross J. Extensive meteoroid fragmentation in V/UHF radar meteor observations at Arecibo Observatory. Geophys. Res. Lett. 2010, vol. 37, no. 4, L04103. DOI: 10.1029/2009GL041967.

McKinley D.W.R. Meteor Science and Engineering. New York, McGraw-Hill, 1961, 309 p.

Nakamura T., Tsuda T., Tsutsumi M., Kita K., Uehara T., Kato S., Fukao S. Meteor wind observations with the MU radar. Radio Sci. 1991, vol. 26, no 04, pp. 857-869. DOI: 10.1029/91RS01164.

Parris R.T. Design and Implementation of a Meteor Tracking Retrofit for the HF Radar at Kodiak Island, Alaska. Thesis (M. S.) University of Alaska Fairbanks, 2003, 123 p.

Premkumar B., Reddy K.C., Yellaiah G., Kumar K.K. Seasonal variations in vertical distribution of meteor decay time as observed from meteor radars at $8.5^{\circ} \mathrm{N}$ and $80^{\circ} \mathrm{N}$. $A d v$. Space Res. 2019, vol. 63, no..5, pp. 1661-1669. DOI: 10.1016/ j.asr.2018.11.019.

Ribeiro A.J., Ruohoniemi J.M., Ponomarenko P.V., Clausen L.B.N., Baker J.B.H., Greenwald R.A., Oksavik, K., de Larquier S. A comparison of SuperDARN ACF fitting methods. Radio Sci. 2013, vol. 48, no. 3, pp. 274-282. DOI: $10.1002 /$ rds. 20031 .

Shepherd S.G. Elevation angle determination for SuperDARN HF radar layouts. Radio Sci. 2017, vol. 52, no. 8, pp. 938-950. DOI: 10.1002/2017RS006348.

Szasz C., Kero J., Meisel D.D., Pellinen-Wannberg A., Wannberg G., Westman A. Orbit characteristics of the tristatic EISCAT UHF meteors. Mon. Not. Royal Astron. Soc. 2008, vol. 388, no. 1, pp. 15-25. DOI: 10.1111/j.1365-2966.2008. 13374.x.

Thomas R.M., Whitham P.S., Elford W.G. Response of high frequency radar to meteor backscatter. J. Atmos. Terr. Phys. 1988, vol. 50, pp. 703-724. DOI: 10.1016/0021-9169(88)90034-7.

Tsutsumi M., Nakamura T., Holdsworth D., Reid I. Meteor observations with an MF radar. Earth, Planets and Space. 1999, vol. 51, no. 7, pp. 691-699. DOI: 10.1186/ BF03353227.

Tsutsumi M., Yukimatu A.S., Holdsworth D.A., Lester M. Advanced SuperDARN meteor wind observations based on raw time series analysis technique. Radio Sci. 2009, vol. 44, no. 2, RS2006. DOI: 10.1029/2008rs003994.

Yukimatu A.S., Tsutsumi M. A new SuperDARN meteor wind measurement: Raw time series analysis method and its application to mesopause region dynamics. Geophys. Res. Lett. 2002, vol. 29, no. 20, pp. 42-1-42-4. DOI: 0.1029/ 2002 GL015210.

Zhu Q., Dinsmore R., Gao B., Mathews J.D. Highresolution radar observations of meteoroid fragmentation and flaring at the Jicamarca Radio Observatory. Mon. Not. Royal Astron. Soc. 2016, vol. 457, no.2, pp. 1759-1769. DOI: $10.1093 / \mathrm{mnras} / \mathrm{stw} 070$. 2020)

URL: http://ckp-rf.ru/ckp/3056 (accessed November 20,

How to cite this article

Fedorov R.R., Berngardt O.I. Monitoring observations of the meteor echo at EKB ISTP SB RAS radar: Algorithms, validation, statistics. Solar-Terrestrial Physics. 2021. Vol. 7. Iss. 1. P. 47-58. DOI: 10.12737/stp-71202107. 